\title{
A GRAPH AND ITS COMPLEMENT WITH SPECIFIED PROPERTIES V: THE SELF-COMPLEMENT INDEX
}

\author{
JIN AKIYAMA, GEOFFREY EXOO AND FRANK HARARY
}

Abstract. The self-complement index $s(G)$ of a graph $G$ is the maximum order of an induced subgraph of $G$ whose complement is also induced in $G$. This new graphical invariant provides a measure of how close a given graph is to being selfcomplementary. We establish the existence of graphs $G$ of order $p$ having $s(G)=n$ for all positive integers $n<p$. We determine $s(G)$ for several families of graphs and find in particular that when $G$ is a tree, $s(G)=4$ unless $G$ is a star for which $s(G)=2$.

\$1. The self-complement index and the induced number. Our purpose is to propose invariants which, in some sense, measure the degree to which a graph is selfcomplementary. To this end we define two related invariants which satisfy this requirement. We then show that the two are equivalent. Throughout we use the notation and terminology of [2]. In particular, all graphs are finite, without loops or multiple lines. The order of a graph $G$ is the number $p$ of points in it. And if $X$ is a set of points in a graph $G$ then we use $\langle X\rangle$ to denote the subgraph of $G$ induced by $X$.

The self-complement index of a graph $G$, denoted $s(G)$, is defined as the order of the largest induced subgraph $H$ of $G$, such that $\bar{H}$ is also induced in $G$. For a graph $G$ of order $p$ it is clear that $1 \leqslant s(G) \leqslant p$ as we do not include the null graph in the family of graphs; see Figure 1 in [3].

Now a related invariant of a graph $G$ is defined. The induced number $m(G)$ is the minimum order of a graph which contains both $G$ and $\bar{G}$ as induced subgraphs.

The first result indicates that $s(G)$ and $m(G)$ are essentially identical. We then proceed to show that $s(G)$ partitions the graphs of order $p$ into $p$ classes which are nonempty except when $s(G)=p$ and $p \equiv 2$ or $3(\bmod 4)$. The number $s(G)$ is then derived for several important families of graphs.

\$2. The equivalence of the two invariants.

THEOREM 1. If $G$ is a graph of order $p$ with self-complement index $s(G)$ and induced number $m(G)$, then

$$
m(G)=2 p-s(G)
$$

Proof. Let $s=s(G), m=m(G)$, and let $H$ of order $s$ be a largest induced subgraph of $G$ whose complement is also induced in $G$.

To prove the upper bound, we construct a graph $F$ of order $2 p-s$ in which both $G$ and $\bar{G}$ are induced subgraphs. Consider disjoint copies of $G$ and $\bar{G}$. Let $U \subset V(G)$

J. Akiyama was a Visiting Scholar, 1978-1979, from Nippon Ika University, Kawasaki, Japan.

[MATHEMATIKA, 27 (1980), 64-68] 
induce $H$ in $G$ and let $W \subset V(\bar{G})$ induce $H$ in $\bar{G}$. Let $\phi: U \rightarrow W$ be a bijection which gives an automorphism of $H$. Then $F$ is obtained from $G \cup \bar{G}$ by identifying each $u \in V$ with $\phi(u)$ in $W$. The order of $F$ is $2 p-s$, and both $G$ and $\bar{G}$ are induced subgraphs of $F$, so $m \leqslant 2 p-s$.

To show the lower bound, suppose $F^{\prime}$ is a graph in which both $G$ and $\bar{G}$ are induced subgraphs. Let $X$ be a set of points of $F^{\prime}$ inducing $G$ and let $Y$ be a set of points inducing $\tilde{G}$. Now if $H^{\prime}$ is the subgraph of $F^{\prime}$ induced by $X \cap Y$, then $H^{\prime}$ is also an induced subgraph of $G$ since $X \cap Y \subset X$. Observe that $\overline{H^{\prime}}$ is also an induced subgraph of $G$. Then because the order of $H$ (a largest induced subgraph of $G$ whose complement is also induced in $G)$ is $s$, we have $|X \cap Y| \leqslant s$. Thus, $m \geqslant|X \cap Y| \geqslant 2 p-s$, as required.

§3. The partition of graphs of order $p$ by $s(G)$. We shall develop an existence theorem for graphs with given self-complement index by showing that for all $p$ and all $n<p$ there exists a graph $G$ of order $p$ with $s(G)=n$. Figure 1 illustrates such graphs for $p=8$, using graph theoretic notation from [3, Ch. 2].

$s=1, \quad m=15$

$$
s=2, \quad m=14
$$

$$
s=3, \quad m=13
$$

$s=4, \quad m=12$

$K_{8}$

$$
K_{4,4}
$$

$K_{7} \cdot K_{2}$

$s=5, \quad m=11$

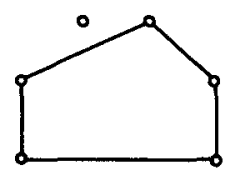

- 0 $s=6, \quad m=10$

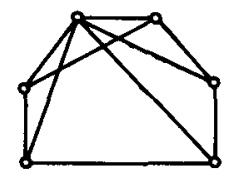

- 0

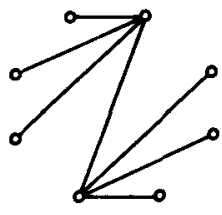

$s=8, \quad m=8$

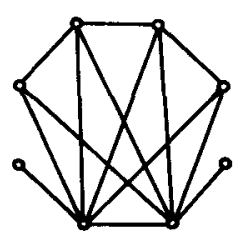

Fig. 1. Examples of 8-point graphs for each possible self-complement index and induced number.

It is well known that self-complementary graphs exist, if, and only if, $p \equiv 0$ or 1 $(\bmod 4)$. Thus in our terminology there exist graphs of order $p$ with self-complement index $p$, if, and only if, $p \equiv 0$ or $1(\bmod 4)$.

Moreover the following properties follow immediately from the definition of selfcomplement index, and so the proofs are omitted.

THEOREM 2. Let $G$ be a graph of order $p$.

(1) $s(G)=s(\bar{G})$.

(2) $s(G)=p$, if, and only if, $G$ is self-complementary.

(3) If $H$ is a maximal induced self-complementary subgraph of $G$, then $s(G) \geqslant|V(H)|$. 
We shall find it convenient to use the following ternary and the quaternary operations which were introduced in [1] and [2]. Following the notation and terminology of [3], the join $G_{1}+G_{2}$ of two graphs is the union of $G_{1}$ and $G_{2}$ with the complete bigraph having point sets $V_{1}$ and $V_{2}$, and the corona $G_{1} \circ G_{2}$ of two graphs $G_{1}$ with $p$ points $v_{i}$ and, $G_{2}$ is obtained from $G_{1}$ and $p$ copies of $G_{2}$ by joining each point $v_{i}$ of $G_{1}$ with all the points of the $i$-th copy of $G_{2}$. We shall require two related ternary operations denoted $G_{1}+G_{2}+G_{3}$ and $G_{1}+G_{2} \circ G_{3}$. The ternary operation written $G_{1}+G_{2}+G_{3}$ on three disjoint graphs is defined as the union of the two joins $G_{1}+G_{2}$ and $G_{2}+G_{3}$. On the other hand, the ternary operation $G_{1}+G_{2} \circ G_{3}$ is defined as the union of the join $G_{1}+G_{2}$ with the corona $G_{2} \circ G_{3}$. Thus this resembles the composition of the path $P_{3}$, not with just one other graph, but with three graphs, one for each point of the path. Figure 2a illustrates the "random" graph $K_{4}-e=K_{1}+K_{2}+K_{1}$ and Figure $2 \mathrm{~b}$ illustrates the graph $A=K_{1}+K_{2} \circ K_{1}$. Of course, the quaternary operation $G_{1}+G_{2}+G_{3}+G_{4}$ is defined similarly and Figure 2c shows the graph $K_{1}+C_{5}+K_{1}+K_{1}$ which will occur in the proof of Theorem 3 .

(a)

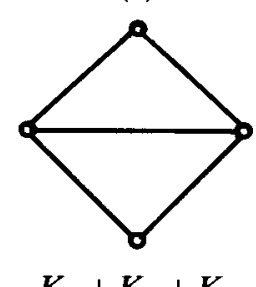

(b)

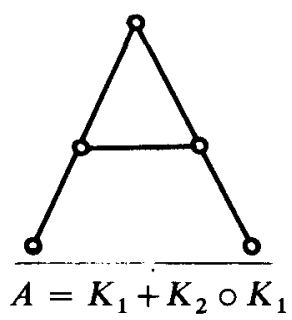

(c)

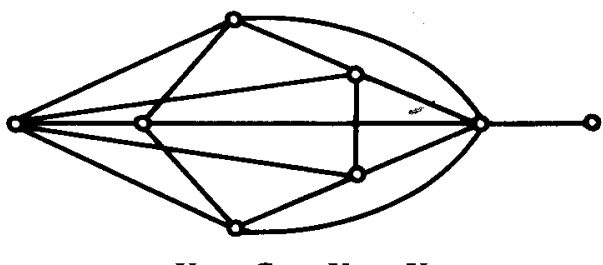

Fig. 2. Examples illustrating the ternary and quaternary operations.

THeORem 3. For all $p$ and all positive integers $n<p$, there exists a graph $G$ of order $p$ with $s(G)=n$.

Proof. We consider four cases according to whether $n \equiv 0,1,2$ or $3(\bmod 4)$.

Case $0 . \quad n \equiv 0(\bmod 4)$. Let $H$ be a self-complementary graph of order $n$. Then if $G=H \cup \bar{K}_{p-n}$, we have $s(G)=n$.

Case $1 . \quad n \equiv 1(\bmod 4)$. This case is handled exactly as Case 0 .

Case 2. $n \equiv 2(\bmod 4)$. Let $H$ be a self-complementary graph of order $n-1$ and let $G=\left(H+K_{1}\right) \cup \bar{K}_{p-n}$. Then $s(G)=n$ since $H+K_{1}$ and $H \cup K_{1}=\overline{H+K_{1}}$ are maximal complementary induced subgraphs of $G$. The maximality follows, since any larger induced subgraph $H^{\prime}$ contains either two isolated points or is $\left(H+K_{1}\right) \cup K_{1}$. But, if $H^{\prime}$ contained two isolated points, then $\overline{H^{\prime}}$ would have two points of degree $d \geqslant n-1$. But $G$ does not have two such points. On the other hand, if $H^{\prime}=\left(H+K_{1}\right) \cup K_{1}$, it is easy to verify that $\overline{H^{\prime}}$ is not induced in $G$.

Case 3. $n \equiv 3(\bmod 4)$. Let $H$ be a self-complementary graph of order $n-2$. Define the graph $G$ to be $\left(K_{1}+H+K_{1}+K_{1}\right) \cup \bar{K}_{p-n-1}$. It now follows that $s(G)=n$ 
since $H+K_{1}+K_{1}$ and $\left(H+K_{1}\right) \cup K_{1}=\overline{H+K_{1}+K_{1}}$ are maximal complementary induced subgraphs of $G$. The maximality of the induced subgraph $H+K_{1}+K_{1}$ of order $n$ is easily verified as in the preceding case.

\$4. The self-complement index of some families of graphs. We now derive the selfcomplement index for several important families of graphs. It is convenient to write $G>H$ when $H$ is an induced subgraph of $G$.

THEOREM 4. The self-complement indexes of complete graphs, complete bigraphs, complete graphs plus one endline, cycles, and complete graphs plus two independent endlines, all of order $p$, are given by:

(1) $s\left(K_{p}\right)=1$;

(2) $s\left(K_{m, n}\right)=2, \quad m+n=p$ and $\max \{m, n\} \geqslant 2$;

(3) $s\left(K_{p-1} \cdot K_{2}\right)=3$;

(4) $s\left(C_{p}\right)=4$;

(5) $s\left(K_{p-4}+K_{2} \circ K_{1}\right)=5, p \geqslant 5$.

Proof. (1) By Theorem 2(3), $s(G) \geqslant 1$ for all graphs $G$ since $K_{1}$ is trivially an induced subgraph of any graph.

Conversely, if $s(G) \geqslant 2$, then $G$ must have two non-adjacent points, and since any pair of points of $K_{p}$ are adjacent, $s\left(K_{p}\right)=1$.

(2) Let $G$ be a complete bigraph $K_{m, n}$. Since $G$ contains both $K_{2}$ and $\bar{K}_{2}$ as induced subgraphs, $s(G) \geqslant 2$. Let $H$ be a graph of order 3 and assume that $G>H$ and $G>\bar{H}$. Since $H$ or $\bar{H}$ contains $P_{3}$ or $K_{3}$ as an induced subgraph, $G>P_{3}$ and $G>\bar{P}_{3}=K_{2} \cup K_{1}$, or $G>K_{3}$ and $G>\bar{K}_{3}$. However, both cases are impossible for $K_{m, n}$.

(3) Let $G$ be a graph $K_{p-1} \cdot K_{2}$. Since $G$ contains $P_{3}$ and $\bar{P}_{3}=K_{2} \cup K_{1}$ as induced subgraphs, $s(G) \geqslant 3$. Let $H$ be a graph of order 4 and assume that $G>H$ and $G>\bar{H}$. Either $H$ or $\bar{H}$ must contain as an induced subgraph one of the following: $\bar{K}_{4}, K_{2} \cup \bar{K}_{2}, P_{3} \cup K_{1}, 2 K_{2}, K_{1,3}$ or $P_{4}$. Therefore, $G$ must also contain one of them as an induced subgraph, which is impossible for $G=K_{p-1} \cdot K_{2}$ since $G$ contains neither $\bar{K}_{3}$ nor $2 K_{2}$ as an induced subgraph.

(4) If $p \geqslant 6$, the cycle $C_{p}$ contains $P_{4}$ as an induced subgraph and so $s\left(C_{p}\right) \geqslant 4$. For any 5 points $W$ of $C_{p}$, the graph $\overline{\langle W\rangle}$ contains $C_{3}$, a contradiction as no larger cycle contains a triangle.

(5) Let $G$ be the graph $K_{p-4}+K_{2} \circ K_{1}, p \geqslant 5$. Since $G$ contains a selfcomplementary graph $A=K_{1}+K_{2} \circ K_{1}$ of order 5 as an induced subgraph, we have $s(G) \geqslant 5$. If $G$ contains an induced subgraph $H$ of order 6 such that $\bar{H}$ is also induced in $G$, then either $H$ or $\bar{H}$ contains at most seven lines since $\left(\begin{array}{l}6 \\ 2\end{array}\right)=15$. But the subgraph induced by any 6 points of $G$ contains at least 8 lines. Thus $s(G)=5$.

By Theorem 4(2), every star has self-complement index 2 . We now determine this index for all other trees. 
TheOREM 5. For any tree $T$ other than a star,

$$
s(T)=4
$$

Proof. Note that all trees with at most 3 points are stars. Unless $T$ is a star, $T$ contains $P_{4}$ as an induced subgraph, so that $s(T) \geqslant 4$. Now we show the reverse inequality. Assume that $s(T) \geqslant 5$, that is, there exists a subtree $H$ of $G$ of order 5 such that $T>H$ and $T>\bar{H}$. This is impossible since $\bar{H}$ must then contain a cycle.

\section{References}

1. J. Akiyama and F. Harary. "A graph and its complement with specified properties III: Girth and circumference", Int'l J. Math. and Math. Sci., 2 (1979), 685-692.

2. J. Akiyama and F. Harary. "A graph and its complement with specified properties IV: Counting selfcomplementary blocks", J. Graph Theory, to appear.

3. F. Harary. Graph theory (Addison-Wesley, Reading, Massachusetts, 1969).

4. F. Harary and R. C. Read. "Is the null graph a pointless concept?" Graphs and combinatorics (R. Bari and F. Harary, eds.), Springer Lecture Notes, 406 (1974), 37-44.

University of Michigan, Department of Mathematics,

Ann Arbor, M1 48109, U.S.A.
05C99: COMBINATORICS; Graph theory.

Received on the 11th of December, 1979. 